\title{
Effects of Safinamide on Pain in Fluctuating Parkinson's Disease Patients: A Post-Hoc Analysis
}

\author{
Carlo Cattaneo ${ }^{\mathrm{a}, *}$, Paolo Barone ${ }^{\mathrm{b}}$, Erminio Bonizzoni ${ }^{\mathrm{c}}$ and Marco Sardina ${ }^{\mathrm{d}}$ \\ ${ }^{a}$ Medical Department, Zambon SpA, Bresso (MI), Italy \\ ${ }^{\mathrm{b}}$ Centre for Neurodegenerative Diseases (CEMAND), Department of Medicine and Surgery, \\ University of Salerno, Baronissi (SA), Italy \\ ${ }^{\mathrm{c}}$ Department of Clinical Science and Community, Section of Medical Statistics and Biometry \\ "GA Maccacaro", University of Milan, Milan, Italy \\ ${ }^{\mathrm{d}} R \& D$ Department, Zambon SpA, Bresso (MI), Italy
}

Accepted 20 September 2016

\begin{abstract}
.
Background: Pain, a frequent non-motor symptom in Parkinson's Disease (PD), significantly impacts on quality of life. Safinamide is a new drug with dopaminergic and non-dopaminergic properties, approved in Europe as adjunct therapy to levodopa for the treatment of fluctuating PD patients. Results from two 24-month, double-blind, placebo-controlled studies demonstrated that safinamide has positive effects on both motor functions and quality of life in PD patients.

Objective: To investigate the effects of safinamide on pain management in PD patients with motor fluctuations using pooled data from studies 016 and SETTLE.

Methods: This post-hoc analysis evaluated the reduction of concomitant pain treatments and the changes in the scores of the items related to pain of the Parkinson's Disease Quality of Life Questionnaire (PDQ-39). A path analysis was performed in order to examine direct and indirect associations between safinamide and PDQ-39 pain-related items assessed after 6-months of treatment.

Results: The percentage of patients with no pain treatments at the end of the trials was significantly lower in the safinamide group compared to the placebo group. Safinamide $100 \mathrm{mg} /$ day significantly reduced on average the individual use of pain treatments by $\approx 24 \%$ and significantly improved two out of three PDQ-39 pain-related items of the "Bodily discomfort" domain.

Path analysis showed that the direct effect of safinamide on pain accounted for about $80 \%$ of the total effect.

Conclusions: These results suggest that safinamide may have a positive effect on pain, one of the most underestimated non-motor symptoms. Prospective studies are warranted to investigate this potential benefit.
\end{abstract}

Keywords: Safinamide, glutamate, pain, Parkinson's disease

\section{INTRODUCTION}

${ }^{*}$ Correspondence to: Carlo Cattaneo, DBSc CAAN, Corporate Medical Advisor CNS, Zambon SpA, Via Lillo del Duca 10, 20091 Bresso (Milan), Italy. Tel.: +39 02 66524299/+39 347 6174245; Fax:+3902 66524887; E-mail: carlo.cattaneo@zambon group.com.

Parkinson's Disease (PD) is generally associated with characteristic motor symptoms (resting tremor, rigidity, bradykinesia and postural instability), caused by the degeneration of the dopaminergic nigrostriatal 
cells [1]. However, patients with PD also experience many non-motor symptoms (NMS), that might be at least as debilitating as the movement disorders and have a strong impact on patients' quality of life [2].

The pathophysiology of NMS is still poorly understood, and a dysfunction of both dopaminergic and non-dopaminergic systems contributes to their development. Drugs that focus only on the dopaminergic system are unable to alleviate non-motor symptoms, while agents that interact with several neurotransmission systems might be very helpful for the treatment of PD [3].

Pain is an important non-motor symptom of $\mathrm{PD}$, often underestimated and inadequately treated [4]. The neurobiology of pain in PD is complex and appears to involve serotonergic, noradrenergic, glutamatergic and gamma-aminobutyric acid (GABA)-ergic neurotransmission, in addition to the dopaminergic systems [5].

Safinamide is a new compound with a unique dual mechanism of action (dopaminergic and nondopaminergic), recently approved by the European Medicines Agency (EMA) for the treatment of midto late-stage fluctuating PD patients as add-on therapy to levodopa (alone or in combination with other antiparkinson drugs).

As safinamide has been shown to inhibit state- and use-dependent sodium channels [6] and since sodium channels inhibitors have been shown to improve neuropathic pain [7, 8], a post-hoc analysis of the pooled data from trials 016 and SETTLE was performed to evaluate the effects of safinamide $100 \mathrm{mg}$ /day on pain management in PD patients with motor fluctuations.

\section{MATERIALS AND METHODS}

\section{Patients}

Studies 016 (NCT01187966) [9] and SETTLE (NCT00627640) [10-12] were phase III, 24-week, double blind, placebo-controlled, parallel-group, randomized, multicenter and multinational trials designed to evaluate the efficacy and safety of safinamide compared to placebo as add-on therapy to stable doses of levodopa (alone or with other antiparkinson drugs) in patients with mid- to latestage PD and motor fluctuations.

Study 016 was conducted in 52 centers in 3 countries, while study SETTLE was conducted in 126 centers in 21 countries. Baseline demographics and clinical characteristics were similar between studies (Table 1). Both protocols and patient materials were approved by Independent Ethics Committees and Health Authorities in all the participating countries. All patients signed an informed consent form and the studies were conducted according to the Declaration of Helsinki.

Key inclusion criteria were: male or female patients, age 30 to 80 years, a diagnosis of idiopathic PD of $\geq 3$ years duration, Hoehn and Yahr stage I-IV during OFF time and motor fluctuations ( $>1.5$ hours' OFF time/day). Key exclusion criteria were: patients with late-stage PD experiencing severe, disabling peak-dose or biphasic dyskinesia, or unpredictable or widely swinging symptom fluctuations, evidence of dementia, major psychiatric illnesses, severe and progressive medical illnesses.

Table 1

Baseline patient demographics and clinical characteristics

\begin{tabular}{|c|c|c|c|c|}
\hline \multirow[t]{2}{*}{ Characteristic } & \multicolumn{2}{|c|}{ Study 016} & \multicolumn{2}{|c|}{ Study SETTLE } \\
\hline & $\begin{array}{l}\text { safinamide } \\
100 \mathrm{mg} / \text { day } \\
(n=224)\end{array}$ & $\begin{array}{l}\text { placebo } \\
(n=222)\end{array}$ & $\begin{array}{l}\text { safinamide } \\
100 \mathrm{mg} / \text { day } \\
(n=274)\end{array}$ & $\begin{array}{l}\text { placebo } \\
(n=275)\end{array}$ \\
\hline Age, years, mean (SD) & $60.1(9.2)$ & $59.4(9.4)$ & $61.7(9.0)$ & $62.1(8.9)$ \\
\hline H\&Y stage, mean (SD) & $2.8(0.6)$ & $2.8(0.7)$ & $2.5(0.6)$ & $2.5(0.6)$ \\
\hline Disease duration, years, mean (SD) & $8.2(3.8)$ & $8.3(3.8)$ & $8.9(4.3)$ & $8.9(4.6)$ \\
\hline Daily OFF time, hours, mean (SD) & $5.3(2.1)$ & $5.2(2.2)$ & $5.3(2.0)$ & $5.4(2.0)$ \\
\hline $\begin{array}{l}\text { Daily ON time with no/non-troublesome } \\
\text { dyskinesia, hours, mean (SD) }\end{array}$ & $9.5(2.4)$ & $9.3(2.2)$ & $9.3(2.4)$ & $9.1(2.5)$ \\
\hline UPDRS III score, mean (SD) & $28.3(13.3)$ & $28.7(12.0)$ & $22.4(11.8)$ & $23.2(12.9)$ \\
\hline UPDRS IV score, mean (SD) & $5.6(2.7)$ & $5.6(2.8)$ & $5.9(2.9)$ & $5.9(2.8)$ \\
\hline GRID-HAMD total score, mean (SD) & $6.0(3.5)$ & $5.9(3.7)$ & $4.7(4.0)$ & $5.0(4.1)$ \\
\hline PDQ-39 BD score, mean (SD) & $28.0(21.4)$ & $28.8(22.0)$ & $36.1(22.9)$ & $34.2(21.9)$ \\
\hline L-dopa dose, mg/day, mean (SD) & $579.6(310.0)$ & $618.5(335.7)$ & $760.8(445.9)$ & $792.3(400.7)$ \\
\hline
\end{tabular}

GRID-HAMD = Grid version of the Hamilton Rating Scale for Depression; H\&Y=Hoehn and Yahr; L-dopa =levodopa; $n=$ number of patients; PDQ-39 BD=Parkinson's Disease Questionnaire 39 items; Bodily discomfort domain; SD=standard deviation; UPDRS III = Unified Parkinson's Disease Rating Scale part III; UPDRS IV = Unified Parkinson's Disease Rating Scale part IV. 


\section{Treatments}

In study 016 patients were randomized to safinamide $50 \mathrm{mg} /$ day, $100 \mathrm{mg} /$ day or placebo, while in the SETTLE trial patients in the safinamide group started at $50 \mathrm{mg} /$ day and after two weeks increased the dose to $100 \mathrm{mg} /$ day. For this reason in the present post-hoc analysis we have considered only the $100 \mathrm{mg}$ dose. The doses of levodopa and other PD treatments were optimized during the pre-treatment stabilization phase, with the aim of remaining stable during the treatment period. However, in both studies the dose of levodopa could be increased, or additional PD drugs [except monoamine oxidase-B (MAO-B) inhibitors] could be used as 'rescue medication' if a patient experience deterioration in motor symptoms. In addition, the dose of levodopa could be decreased in response to the patient's condition or the occurrence of adverse events.

Treatments with tri-tetracyclic antidepressants, MAO-B inhibitors, serotonin-norepinephrine reuptake inhibitors (SNRIs), opioids, neuroleptics, barbiturates and phenothiazines were not permitted in both studies. The use of selective serotonin reuptake inhibitors (SSRIs) was allowed, provided that the dose was kept as low as possible and remained stable throughout the trial; dextromethorphan was permitted if used for treating cough.

Pain treatments included analgesics, antiinflammatory, anti-rheumatic drugs and topical products for joint and muscular pain.

\section{Outcome measures}

This is a post-hoc analysis of the pooled data from studies 016 and SETTLE, evaluating the effects of safinamide $100 \mathrm{mg} /$ day oral tablets versus placebo on the reduction of concomitant pain treatments and on the scores of pain-related items of the PDQ-39 "Bodily discomfort" domain. Considering the large number of patients enrolled and the similarity of trial design between the two studies, a post-hoc analysis can provide important and clinically relevant information about the effect of adjunctive safinamide treatment on pain.

A path analysis model was implemented in order to differentiate the direct treatment effects of safinamide on pain reduction from effects mediated indirectly through alleviation of motor symptoms, as recorded on the PDQ-39 "Bodily discomfort" domain assessed after 6-month of treatment. Path analysis is generally designed to test linkages and the causal relations among variables by measuring the contribution of direct and indirect effects of one variable on others, and often used to explore which treatment pathways contribute to the overall efficacy of a drug [13].

The path diagram reported in Fig. 1 depicts the hypothesized relationships among the variables with a single headed arrow representing the causal order between two variables, with the head pointing to the effect (endogenous variable) and the tail to the cause (exogenous variable).

Four endogenous variables were: 6-month OFF time, 6-month Unified Parkinson's Disease Rating Scale part IV (UPDRS IV) score, 6-month Grid version of the Hamilton Rating Scale for Depression (GRID-HAMD) score, and 6-month PDQ-39 "Bodily discomfort" score.

Five exogenous variables were: baseline OFF time, baseline UPDRS IV score, baseline GRID-HAMD score, baseline PDQ-39 "Bodily discomfort" score and randomized treatment (safinamide $100 \mathrm{mg}$ /day or placebo).

OFF time, UPDRS IV and GRID-HAMD were used to determine the indirect effects of safinamide on pain, mediated by the improvements in these variables. Pain, in fact, is known to be associated in PD with motor complications (including motor fluctuations, dystonia and dyskinesia), that are measured by OFF time and UPDRS IV, while no association has been clearly detected with motor symptoms as measured by UPDRS III [14].

Depression (measured by GRID-HAMD) is a variable with a potentially confounding effect on pain [14].

The main assumption of the path analysis was that relations between variables are linear and the effects observed on pain are additive (i.e., the direct plus the indirect effect results in the total effect). Additional assumptions include adequate sample size, low multicollinearity and that error terms should not be correlated to any variable.

\section{Statistical methods}

The comparison of the percentage of patients not using concomitant pain drugs after 6-months treatment was performed using conventional Pearson's Chi-Square. The reduction in the number of pain treatments associated with safinamide $100 \mathrm{mg}$ /day versus placebo was estimated by means of a Negative Binomial regression model obtained with a Generalized Linear Model parameterized with logarithmic link 


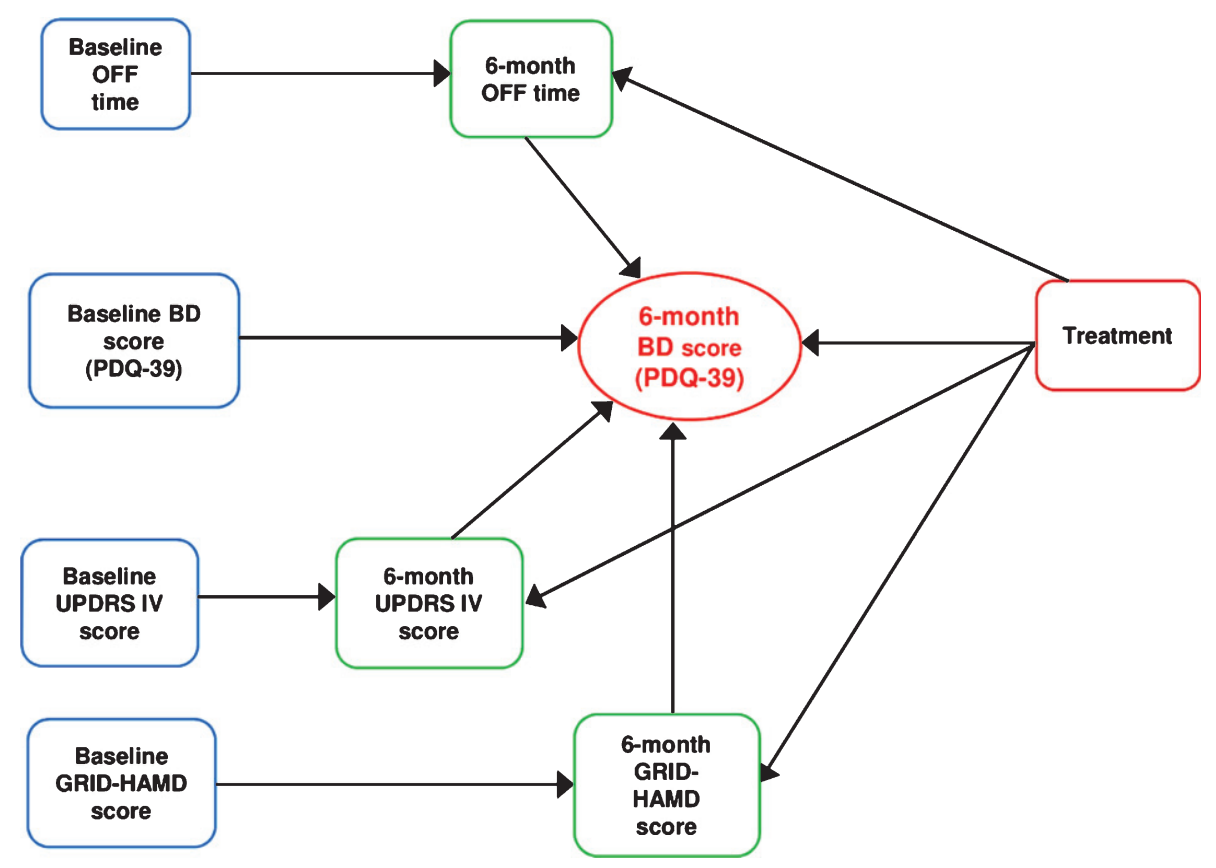

Fig. 1. Path analysis diagram of variables contributing to pain in patients. $\mathrm{BD}=$ Bodily discomfort domain; PDQ-39= Parkinson's Disease Questionnaire 39 items; GRID-HAMD = Grid version of the Hamilton Rating Scale for Depression; UPDRS IV = Unified Parkinson's Disease Rating Scale part IV.

function and negative binomial distribution and with "Treatment" (safinamide $100 \mathrm{mg} /$ day or placebo) and "Study indicator" (016 or SETTLE) as fixed effects. The analysis was performed using the GENMOD Procedure of SAS software version 9.4.

The analyses of PDQ-39 domain "Bodily discomfort" and PDQ-39 individual items related to pain were performed using an ANCOVA model with PDQ-39 scores changes from baseline as dependent variable, with "Treatment" (safinamide $100 \mathrm{mg}$ /day or placebo) and "Study indicator" (016 or SETTLE) as fixed effects and with baseline values as covariate.

Regarding the path analysis, standard procedures were followed to test whether the data fit the theoretical path model and ensuring that conditions were satisfied for unbiased parameter estimation and interpretation of path model fit [15]. Standardized summary of the average covariance residuals [Root Mean Square Error of Approximation (RMSEA)], standardized difference between the observed correlation and the predicted correlation [standardized root mean square residual (SRMSR)], Bentler Comparative Fit Index (BCFI), the proportion of the observed covariance, and Adjusted Goodness of Fit Index (AGFI) were used to evaluate whether a path model meets the modeling requirement [16]. Generally accepted values for fit indices are
RMSEA $<0.10$, SRMSR $<0.08$, BCFI $>0.90$, and AGFI $>0.90$. In our analysis the values were the following: RMSEA $=0.07$, SRMSR $=0.04$, BCFI $=0.94$ and $\mathrm{AGFI}=0.95$, confirming the accuracy of the path model used.

The sample size in this study was adequate based on the recommendation by Kline [15] that 10-20 times as many cases as parameters is sufficient for significance testing of path analysis model effects. The path analysis was performed using Proc CALIS of SAS 9.4 (SAS Institute, Cary, NC, USA).

\section{RESULTS}

As previously described, safinamide $100 \mathrm{mg} /$ day increased $\mathrm{ON}$ time with no/non-troublesome dyskinesia and improved the secondary endpoints related to motor function, quality of life and OFF time. The incidence of treatment-emergent adverse events and serious adverse events was similar in safinamide and placebo groups [9, 11, 12].

After 24 weeks, the proportion of patients not using concomitant pain treatments was significantly greater in the group receiving safinamide $100 \mathrm{mg}$ /day than in the placebo group (Fig. 2), with a reduction of the number of concomitant pain treatments of 
$23.6 \%$ [95\% confidence interval (CI): $41.1 \%, 1.0 \%$; $p=0.0421]$ compared with placebo.

Moreover, safinamide $100 \mathrm{mg} /$ day significantly improved 2 of the 3 PDQ-39 items related to musculoskeletal and neuropathic pain (Fig. 3). The difference for the changes from baseline in the overall "Bodily discomfort" domain score was also significant: -5.28 (95\% CI: $-6.78,-3.79$ ) in the safinamide $100 \mathrm{mg} /$ day group compared to -1.59 (95\% CI: -3.09 , $-0-10 ; p=0.0007)$ for the placebo group.

In Table 2 the standardized regression coefficients (path coefficients) with associated standard errors and $P$-values estimated with the path analysis are reported. Contrary to classic multivariable regression models, statistical significance of path coefficients is not essential to validate a path model or to rank the importance of variables in order to decide for their inclusion/exclusion. Nevertheless it is worthy of note that all the path coefficients between the variables included in the model are statistically significant or at least statistically borderline $(p<0.1)$. In particular the association of treatment with 6-month pain (measured by 6-month Bodily discomfort score) still remains statistically significant (path coefficient $=-0.0674, p=0.00761$ ) supporting the hypothesis of a direct effect of safinamide on pain.

The path analysis showed that $79.7 \%$ of pain reduction ascribed to safinamide was attributable to a direct

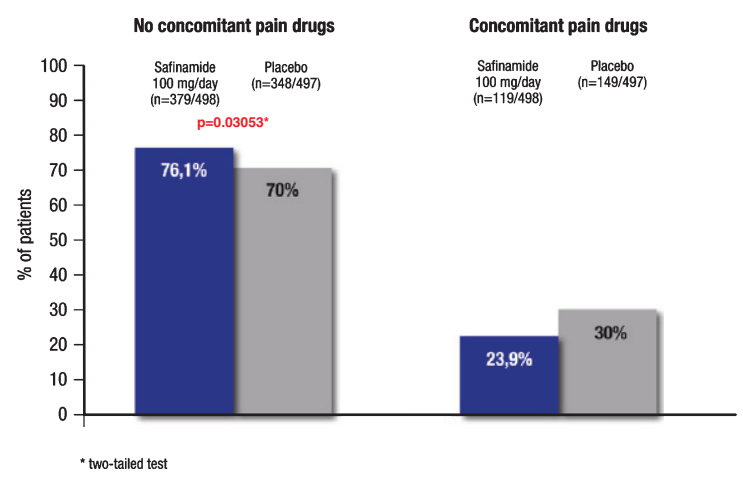

Fig. 2. Trials 016 and SETTLE (pooled data): concomitant use of pain treatments.

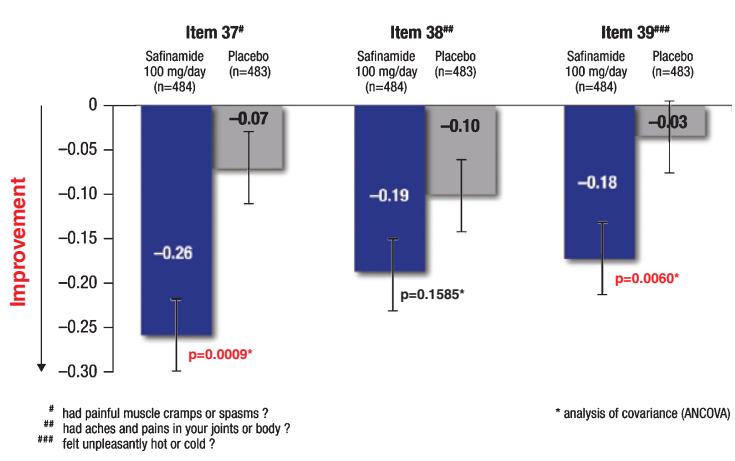

Fig. 3. Trials 016 and SETTLE (pooled data): changes (LS means \pm SE) from baseline to week 24 of the PDQ-39 items 37-39. $\mathrm{LS}=$ least squares; $\mathrm{SE}=$ standard error of the mean.

Table 2

Results of path analysis (pooled data from trials 016 and SETTLE)

\begin{tabular}{|c|c|c|c|c|}
\hline Exogenous variable & Endogenous variable & Path coefficient $(\mathrm{SE})^{\dagger}$ & $p$-value & Interpretation \\
\hline Baseline BD score & 6-month BD score & $0.5698(0.021)$ & 0.00000 & \multirow{4}{*}{$\begin{array}{l}\text { Path coefficients estimating the } \\
\text { magnitude of the effects of } \\
\text { baseline values }\end{array}$} \\
\hline Baseline OFF time & 6-month OFF time & $0.5520(0.022)$ & 0.00000 & \\
\hline Baseline GRID-HAMD score & 6-month GRID-HAMD score & $0.6172(0.020)$ & 0.00000 & \\
\hline Baseline UPDRS IV score & 6-month UPDRS IV score & $0.6834(0.017)$ & 0.00000 & \\
\hline Treatment & 6-month OFF time & $-0.1658(0.026)$ & 0.00000 & \multirow{2}{*}{$\begin{array}{l}\text { The magnitude of the indirect effect } \\
\text { on pain mediated by OFF time is } \\
\text { estimated by multiplying the two } \\
\text { path coefficients }\end{array}$} \\
\hline 6-month OFF time & 6-month BD score & $0.0516(0.025)$ & 0.04092 & \\
\hline Treatment & 6-month GRID-HAMD score & $-0.0451(0.025)$ & 0.07172 & \multirow{2}{*}{$\begin{array}{l}\text { The magnitude of the indirect effect } \\
\text { on pain mediated by GRID-HAMD } \\
\text { is estimated by multiplying the two } \\
\text { path coefficients }\end{array}$} \\
\hline 6-month GRID-HAMD score & 6-month BD score & $0.1017(0.025)$ & 0.00006 & \\
\hline Treatment & 6-month UPDRS IV score & $-0.0394(0.023)$ & 0.09092 & \multirow{2}{*}{$\begin{array}{l}\text { The magnitude of the indirect effect } \\
\text { on pain mediated by UPDRS IV is } \\
\text { estimated by multiplying the two } \\
\text { path coefficients }\end{array}$} \\
\hline 6-month UPDRS IV score & 6-month BD score & $0.1028(0.026)$ & 0.00006 & \\
\hline Treatment & 6-month BD score & $-0.0674(0.025)$ & 0.00761 & Direct effect on pain \\
\hline
\end{tabular}

${ }^{\dagger}$ Path coefficients are standardized regression coefficients ranging from -1 to 1 and measuring the magnitude (importance) of the effect of a variable (exogenous variable) on another one (endogenous variable). A path coefficient equal to 0 means no effect. A path coefficient equal to 1 or to -1 means that the endogenous variable is totally (positively or negatively) predicted by the exogenous variable. BD $=$ Bodily discomfort domain; GRID-HAM-D = Grid version of the Hamilton Rating Scale for Depression; SE = standard error; UPDRS IV = Unified Parkinson's Disease Rating Scale part IV. 


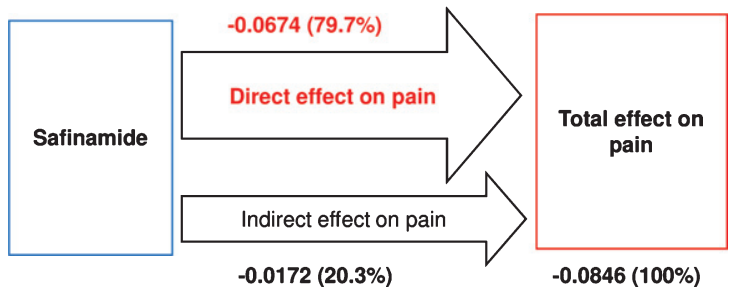

Fig. 4. Path analysis of direct and indirect effects of safinamide on pain. Values represent the path coefficients derived from regression analyses with the proportional contribution to the total treatment effect shown in parentheses.

effect of the drug $(p=0.0076)$, while the remaining $20.3 \%$ was an indirect effect mediated by the its activity on OFF Time (10.1\%), GRID-HAMD (5.4\%) and UPDRS IV (4.5\%) (Fig. 4).

\section{DISCUSSION}

The presence of painful symptoms is well documented in the earliest descriptions of PD [17, 18]. According to epidemiological studies, the prevalence of chronic pain in PD patients is from 30 to $85 \%$ [19, 20]. Pain may precede the full expression of motor symptoms of PD, has been shown to be associated with impaired sleep, depressed mood and reduced health-related quality of life and, in some patients, can be the dominant symptom of PD [21].

The basal ganglia are involved in pain processing, and imbalance of dopaminergic and nondopaminergic systems within the basal ganglia and cortex is believed to contribute to pain in PD [22].

The findings of this post-hoc analysis on the effects of safinamide on pain extended the results obtained in pivotal studies. At week 24, significantly more patients in the safinamide $100 \mathrm{mg} /$ day group were free of pain medications, as compared with placebo group.

The reduction of the number of concomitant pain treatments was about $24 \%$ with safinamide versus placebo, and was associated with significantly greater improvements in 2 of the 3 specific items of the "Bodily discomfort" domain of the PDQ-39 questionnaire, addressing musculoskeletal and neuropathic pain.

Patients with PD frequently report different types of pain, that can be categorized based on their clinical description into musculoskeletal, dystonic, radicular-peripheral neuropathic and central pain [4]. Musculoskeletal and neuropathic pain are the most common: musculoskeletal pain may be related to rigidity, akinesia and pathologic postures such as camptocormia, while neuropathic pain may lead to a decrease of pain threshold [23].

The path analysis, performed to investigate the possibility that the observed improvements may be, at least in part, attributable to improvements in motor complications and mood, showed that the direct effect of safinamide on pain was $79.7 \%$, confirming that the efficacy of the treatment on the pain-related items of the PDQ-39 was not mediated by an alleviation of other symptoms.

These results may be explained by the nondopaminergic mechanism of action of safinamide. In vitro electrophysiological studies have shown that safinamide is a state-dependent inhibitor of human voltage-gated sodium channels (VGSC) in the inactivated state. Deregulation of VGSC expression is thought to be contribute to both chronic inflammation and neuropathic pain [24]. Moreover, dopaminergic denervation in PD leads to the hyperactivity of the glutamatergic pathway, that represents the primary cause of excitotoxicity. Glutamate is released in a calcium and sodium-dependent manner in response to nerve terminal depolarisation. The selective inhibition of glutamatergic hyperactivity may be an effective strategy for the treatment of some PD nonmotor symptoms not responding to L-dopa therapy, in particular pain and neuropsychiatric symptoms [25].

There are several limitations to be considered in this post-hoc analysis: the major limit is that the original trials were not designed to investigate pain as a primary endpoint, so there was no direct evaluation of pain but only indirect measures (quality of life and consumption of pain relief medicines). Another potential bias is the exclusion in the eligibility criteria of concomitant therapies commonly used in routine clinical practice for the treatment of pain in PD (opioids, barbiturates, antidepressants). These analyses should therefore be considered as exploratory, and their clinical relevance must be confirmed in larger clinical trials.

\section{CONCLUSION}

Pain in PD frequently goes overlooked and undertreated in clinical practice.

The loss of dopaminergic neurons causes glutamatergic hyperactivity, the selective inhibition of which could be an effective strategy for the treatment of the PD non-motor symptoms.

Despite the limitations of this post-hoc analysis, the results showed a favorable effect of safinamide 
$100 \mathrm{mg} /$ day on pain, accounting for about $80 \%$ of the total effect, as shown by the path analysis. This effect was aside from the effect on motor fluctuations and dyskinesia, that accounted for about $20 \%$ of the total effect. New prospective studies are warranted to investigate the potential benefits of safinamide on PD pain and their clinical relevance.

\section{ACKNOWLEDGMENTS}

We thank Ray Hill, an independent medical writer, for journal styling prior to submission on behalf of Health Publishing \& Services Srl. The authors have no acknowledgments to disclose.

\section{CONFLICT OF INTEREST}

Paolo Barone is member of the Scientific Advisory Board of Zambon SpA.

Carlo Cattaneo and Marco Sardina are employees at Zambon SpA, the Pharmaceutical Company that is marketing safinamide in Europe.

Erminio Bonizzoni is a consultant statistician for Zambon SpA.

\section{REFERENCES}

[1] Jankovic J (2008) Parkinson's disease: Clinical features and diagnosis. J Neurol Neurosurg Psychiatry, 79, 368-376.

[2] Martinez-Martin P, Rodriguez-Blazquez C, Kurtis MM, \& Chaudhuri KR (2011) The impact of non-motor symptoms on health-related quality of life of patients with Parkinson's disease. Mov Disord, 26, 399-406.

[3] Fox SH (2013) Non-dopaminergic treatments for motor control in Parkinson's disease. Drugs, 73, 1405-1415.

[4] Sophie M, \& Ford B (2012) Management of pain in Parkinson's disease. CNS Drugs, 26, 937-948.

[5] Barone P (2010) Neurotransmission in Parkinson's disease: Beyond dopamine. Eur J Neurol, 17, 364-376.

[6] Caccia C, Maj R, Calabresi M, Maestroni S, Faravelli L, Curatolo L, Salvati P, \& Fariello RG (2006) Safinamide: From molecular targets to a new anti-Parkinson drug. Neurology, 67, S18-S23.

[7] Bhattacharya A, Wickenden AD, \& Chaplan SR (2009) Sodium channel blockers for the treatment of neuropathic pain. Neurotherapeutics, 6, 663-678.

[8] Rogers M, Tang L, Madge DJ, \& Stevens EB (2006) The role of sodium channels in neuropathic pain. Semin Cell Dev Biol, 17, 571-581.

[9] Borgohain R, Szasz J, Stanzione P, Meshram C, Bhatt M, Chirilineau D, Stocchi F, Lucini V, Giuliani R, Forrest E, Rice P, \& Anand R (2014) Randomized trial of safinamide add-on to levodopa in Parkinson's disease with motor fluctuations. Mov Disord, 29, 229-237.
[10] Schapira AH, Fox S, Hauser R, Jankovic J, Kulisevsky J, Pahwa R, Poewe W, von Raison F, Kenney C, \& Musch B (2010) SETTLE study design: A 24-week, double-blind, placebo-controlled study of the efficacy and safety of safinamide as add-on therapy to levodopa in patients with Parkinson's disease [Poster]. Mov Disord, 25, S308.

[11] Anand R, Schapira A, Giuliani R, \& Lucini V (2013) Safinamide is associated with clinically important improvement in motor symptoms in fluctuating PD patients as add-on to levodopa (SETTLE) [Poster]. Mov Disord, 28, S151-S152.

[12] Schapira A, Fox S, Hauser R, Jankovic J, Jost W, Kulisevsky J, Pahwa R, Poewe W, Lucini V, \& Anand R (2013) Safinamide significantly improves responder rates in fluctuating Parkinson's disease (PD) patients as add-on to levodopa (SETTLE) [Poster]. Mov Disord, 28, S152.

[13] Retherford R, \& Choe M (1994) Statistical models for causal analysis. Chapter 4 Path Analysis. John Wiley \& Sons, New York.

[14] Tinazzi M, Del Vesco C, Fincati E, Ottaviani S, Smania N, Moretto G, Fiaschi A, Martino D, \& Defazio G (2006) Pain and motor complications in Parkinson's disease. J Neurol Neurosurg Psychiatry, 77, 822-825.

[15] Kline RB (2010) Principles and Practice of Structural Equation Modeling, 3rd edn Guilford Press, New York.

[16] Browne MW, \& Cudeck R (1992) Alternative ways of assessing model fit. Sociol Methods Res, 21, 230-258.

[17] Parkinson J (1817) An essay on the shaking palsy. Whittingham and Rowland, London.

[18] Charcot JM (1892) Oeuvres completes, Vol. 1 [French] Bureaux du Progres Medical, Paris.

[19] Nègre-Pagès L, Regragui W, Bouhassira D, Grandjean H, Rascol O, \& DoPaMiP Study Group (2008) Chronic pain in Parkinson's disease: The cross-sectional French DoPaMiP survey. Mov Disord, 23, 1361-1369.

[20] Muntean M, \& Perju-Dumbrava L (2009) Pain in Parkinson's disease patients. Rom J Neurol, $8,144$.

[21] Defazio G, Berardelli A, Fabbrini G, Martino D, Fincati E, Fiaschi A, Moretto G, Abbruzzese G, Marchese R, Bonuccelli U, Del Dotto P, Barone P, De Vivo E, Albanese A, Antonini A, Canesi M, Lopiano L, Zibetti M, Nappi G, Martignoni E, Lamberti P, \& Tinazzi M (2008) Pain as a nonmotor symptom of Parkinson disease: Evidence from a case-control study. Arch Neurol, 65, 1191-1194.

[22] Chudler EH, \& Dong WK (1995) The role of the basal ganglia in nociception and pain. Pain, 60, 3-38.

[23] Kassubek J, Chaudhuri KR, Zesiewicz T, Surmann E, Boroojerdi B, Moran K, Ghys L, \& Trenkwalder C (2014) Rotigotine transdermal system and evaluation of pain in patients with Parkinson's disease: A post hoc analysis of the RECOVER study. BMC Neurol, 14, 42.

[24] Salvati P, Maj R, Caccia C, Cervini MA, Fornaretto MG, Lamberti E, Pevarello P, Skeen GA, White HS, Wolf HH, Faravelli L, Mazzanti M, Mancinelli E, Varasi M, \& Fariello RG (1999) Biochemical and electrophysiological studies on the mechanism of action of PNU-151774E, a novel antiepileptic compound. J Pharmacol Exp Ther, 288, 11511159.

[25] Juri C, Rodriguez-Oroz M, \& Obeso JA (2010) The pathophysiological basis of sensory disturbances in Parkinson's disease. J Neurol Sci, 289, 60-65. 